\title{
A Novel Iris Segmentation Scheme
}

\author{
Chen-Chung Liu, ${ }^{1}$ Pei-Chung Chung, ${ }^{2}$ Chia-Ming Lyu, ${ }^{1}$ Jui Liu, ${ }^{3}$ and Shyr-Shen Yu ${ }^{2}$ \\ ${ }^{1}$ Department of Electronic Engineering, National Chin-Yi University of Technology, No. 57, Section 2, Zhongshan Road, \\ Taiping District, Taichung 41170, Taiwan \\ ${ }^{2}$ Department of Computer Science and Engineering, National Chung-Hsing University, 250 Kuo Kuang Road, Taichung 402, Taiwan \\ ${ }^{3}$ Department of Nuclear Medicine, Bankstown-Lidcombe Hospital, Eldridge Road, Bankstown, NSW 2200, Australia
}

Correspondence should be addressed to Shyr-Shen Yu; pyu@nchu.edu.tw

Received 25 February 2014; Accepted 13 April 2014; Published 11 May 2014

Academic Editor: Her-Terng Yau

Copyright (c) 2014 Chen-Chung Liu et al. This is an open access article distributed under the Creative Commons Attribution License, which permits unrestricted use, distribution, and reproduction in any medium, provided the original work is properly cited.

\begin{abstract}
One of the key steps in the iris recognition system is the accurate iris segmentation from its surrounding noises including pupil, sclera, eyelashes, and eyebrows of a captured eye-image. This paper presents a novel iris segmentation scheme which utilizes the orientation matching transform to outline the outer and inner iris boundaries initially. It then employs Delogne-Kåsa circle fitting (instead of the traditional Hough transform) to further eliminate the outlier points to extract a more precise iris area from an eyeimage. In the extracted iris region, the proposed scheme further utilizes the differences in the intensity and positional characteristics of the iris, eyelid, and eyelashes to detect and delete these noises. The scheme is then applied on iris image database, UBIRIS.v1. The experimental results show that the presented scheme provides a more effective and efficient iris segmentation than other conventional methods.
\end{abstract}

\section{Introduction}

Biometric recognition is used to automatically recognize individuals based on the different and unique physiological characteristics such as face, ear, fingerprint, gait, iris, palm prints, and voice. Human's iris pattern can be used as an ideal biometric character due to its uniqueness between individuals and its fixated pattern throughout individual's life $[1,2]$.

The iris recognition scheme consists of six steps: eyeimage capture, iris extraction, normalization, preprocessing, feature extraction, and matching [2]. Accurate iris extraction is probably the most important step because it greatly influences the overall recognition accuracy and processing speed. The iris is often surrounded by noise, such as the pupil, sclera, eyelashes, and eyebrows, which will need to be removed accordingly to achieve accurate iris extraction.

In the published iris segmentation schemes, the integral differential operator and Hough transform are frequently utilized. Daugman [3] used an integral differential operator to remove the potential eyelid noise to localize the iris regions. He used the gradient ascent and radial Gaussian methods to fit the circular contours. De Martin-Roche et al. [4] applied the histogram stretch method on a grayscale eyeimage to find the three circumference parameters, center $(\alpha, \beta)$, and radius $R$ to maximize the average intensity differences of five consecutive circumferences. Circles with the detected circumference parameters are considered the iris-boundaries. Camus and Wildes [5] took a gradient-based Hough transform on the edge map of an eye-image to detect the iris-boundaries with a filtering and voting procedure. This method only performed well when the iris is obviously separated from both the pupil and the sclera. Proença and Alexandre [6] tried four different clustering schemes to preprocess eye-images to enhance their contrast for iris segmentation. They found that the fuzzy $k$-means clustering scheme provides the best performance when it is utilized on the position and intensity feature vector.

To preserve the advantages and overcome the disadvantages of the above-mentioned methods, a novel iris segmentation scheme is proposed. Instead of these popular edge detection schemes and circular Hough transform, the orientation matching transform (OMT) is utilized to find the outer and inner iris-boundaries and then a mathematical 
technique, Delogne-Kåsa circle fitting (DKCF) scheme, is used to eliminate the outlier points of the rough outer and inner iris-boundaries to extract a more precise iris area from an eye-image. Moreover, the proposed scheme utilizes the intensity and positional characteristics of the iris, eyelid, and eyelashes to obtain more accurate iris segmentation.

The performance of the proposed iris segmentation scheme is verified using an iris image database, UBIRIS.v1, created at the Soft Computing and Image Analysis Lab (SOCIA Lab) of the University of Beira Interior. This database consists of a set of visible wavelength noisy iris images, captured at close-up distance with user cooperation [7]. Several error measures, such as misclassification error, relative foreground area error, accuracy, nonuniformity region, and edge mismatch are conducted to measure the performance of the proposed algorithm. The experimental results show that the proposed algorithm provides a more effective and efficient iris segmentation than other methods. The remainder of this paper is organized as follows. Section 2 illustrates the proposed iris extraction scheme. Section 3 presents the experiment results. Conclusions are given in Section 4.

\section{Proposed Scheme}

The proposed scheme is divided into three stages: (i) detecting inner and outer iris-borders, (ii) detecting eyelids, and (iii) detecting eyelashes. Figure 1 shows the flow chart of the proposed scheme.

2.1. Stage 1: Inner and Outer Iris-Border Detecting. The flow chart of the proposed inner and outer iris-border detection is shown in Figure 2. An RGB color human eye-image is inputted and transformed from the RGB domain into the YIQ domain. Since the $y$-component occupies 93\% of the total energy in the YIQ domain, only the $y$-component is used in the following processes to save operating time without degrading the segmentation quality. The iris in an eye-image is an annulus bounded by an inner boundary and an outer boundary. Both the inner and outer boundaries should be located before the iris extraction. Since the inner iris-boundary is the boundary between iris and pupil, to locate the inner boundary is to detect the pupil. The detailed steps for inner and outer iris-border detection are illustrated in the following subsections.

2.1.1. Pupil Detecting Using Outliers Detection. In statistics an outlier is defined as an observation that deviates substantially from the other observations that it is considered generated by a different system. Outliers frequently affect the parameters estimation for finding a model fitting to the data. The outliers are frequently removed to improve the accuracy of the estimators. To define the outliers of a data set, let the mean and let $\bar{x}$ be the standard deviation $\sigma$ of the data set. The observation will be declared as a lower outlier if it is less than $(\bar{x}-k \sigma)$ and as an upper outlier if it is more than $(\bar{x}+k \sigma)$; the value of $k$ is usually taken from the range between 2 and 3 .

In a grayscale eye-image, the darkest areas are the pupil, eyelashes, and shadow regions. These dark regions almost

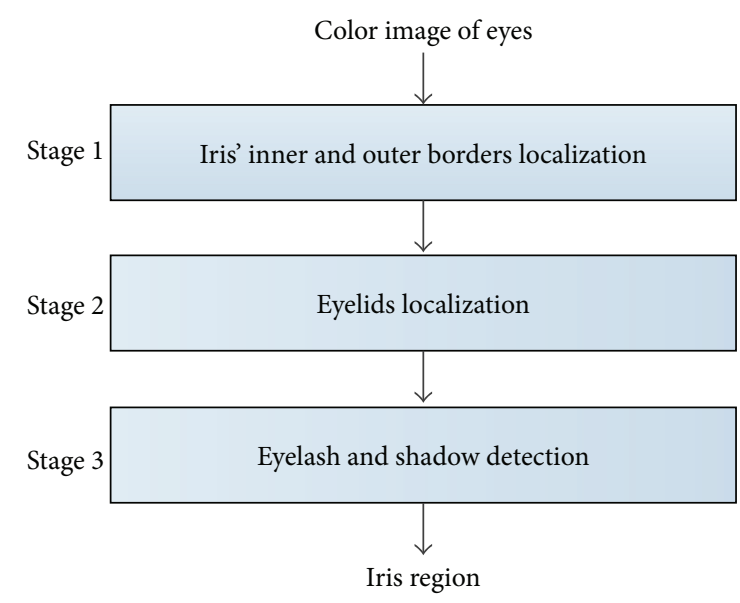

FIgURE 1: The flow chart of the proposed scheme.

always locate in the lower outliers of the pixel value distribution of a grayscale eye-image. It is therefore reasonable to take the lower outlier points of the eye-image as the candidate pupil points. Then the Canny edge filter, OMT, and DKCF are further applied on the candidate pupil regions to extract a more accurate inner iris-boundary.

2.1.2. Circle Detection with Orientation Matching Transform. The Circle Hough Transform (CHT) is a known algorithm for finding given radius circular shapes within an image [8]. The main disadvantages of $\mathrm{CHT}$ are large memory requirements and long computation time. Kimme et al. [9] modified the CHT and proposed the edge orientation based CHT (EOCHT) by utilizing the edge orientation of each element of a circle pointing toward or away from the circle's center. The EOCHT only needs to plot an arc perpendicular to the edge orientation at a distance from the edge point. This reduces the computation cost. Ceccarelli et al. [10] utilized the edge orientation characteristic of circle's elements to develop the Orientation Matching Transform (OMT) for detecting circles with radii in the range $\left[R_{m}, R_{M}\right]$. It is illustrated as follows.

Let $f(x, y)$ be an image and let $\hat{f}(x, y)=\nabla f(x, y) /$ $|\nabla f(x, y)|=\langle\cos \varphi, \sin \varphi\rangle$ be the orientation unit-vector of image gradient at pixel $(x, y)$, where

$$
\begin{aligned}
\cos \varphi & =\frac{(\partial f / \partial x)}{\sqrt{(\partial f / \partial x)^{2}+(\partial f / \partial y)^{2}}} \\
\sin \varphi & =\frac{(\partial f / \partial y)}{\sqrt{(\partial f / \partial x)^{2}+(\partial f / \partial y)^{2}}}
\end{aligned}
$$

Let $A_{R_{m}}^{R_{M}}(\alpha, \beta)$ be the annulus within the image $f$, with inner radius $R_{m}$, outer radius $R_{M}$, and center $(\alpha, \beta)$ defined as

$$
\begin{aligned}
& A_{R_{m}}^{R_{M}}(\alpha, \beta) \\
& =\left\{(x, y) \in f \mid R_{m}^{2} \leq(x-\alpha)^{2}+(y-\beta)^{2}\right. \\
& \left.\quad \leq R_{M}^{2}, x \in \mathfrak{R}, y \in \mathfrak{R}\right\},
\end{aligned}
$$




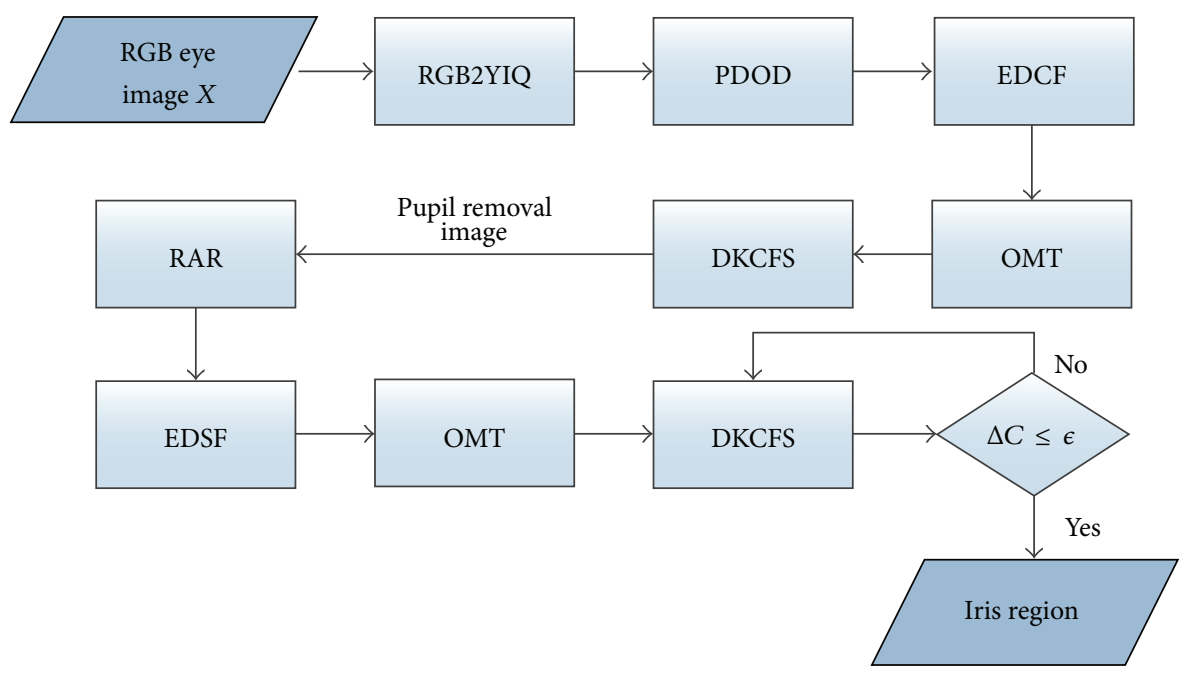

RGB2YIQ: color mapping RGB to YIQ

PDOD: pupil detection using outliers detection

EDCF: edge detection using Canny filter

OMT: orientation matching transform

DKCFS: Delogne-Kåsa circle fitting scheme

RAR: redundant areas removal

EDSF: edges detection using Sobel filter

$\Delta C$ : absolute difference between presented and last circles

FIgURE 2: The flow chart of the proposed iris detection algorithm.

$$
\begin{aligned}
& \operatorname{OMT}(\alpha, \beta) \\
& =\iint_{A_{R_{m}}^{R_{M}}(x, y)} \frac{(\hat{f}(x, y) \cdot \vec{g}(x-\alpha, y-\beta)) d x d y}{\left(2 \pi\left(R_{M}-R_{m}\right)\right)},
\end{aligned}
$$

where $\vec{g}(x-\alpha, y-\beta)$ is the kernel vector (orientation vector of points of circles with center $(\alpha, \beta)$ defined as follows:

$$
\begin{aligned}
\vec{g}(x- & \alpha, y-\beta) \\
& =\left(\frac{\cos \left(\tan ^{-1}(y / x)\right)}{\sqrt{(x-\alpha)^{2}+(y-\beta)^{2}}}, \frac{\sin \left(\tan ^{-1}(y / x)\right)}{\sqrt{(x-\alpha)^{2}+(y-\beta)^{2}}}\right) .
\end{aligned}
$$

The OMT is also used to evaluate how many points in an image have the gradient orientation coincident with the gradient orientation of circles with radii in the range $\left[R_{m}, R_{M}\right]$ and uses an accumulator to find the most likely coordinates of a circle in the image. The peak in the accumulator array denotes the candidates of the circle center in the image. Once the center $(\alpha, \beta)$ is decided, the points located in $A_{R_{m}}^{R_{M}}(\alpha, \beta)$ are collected as the candidate points of the pupil boundary. Because iris images in UBIRIS.v1 were captured at close-up distance with user cooperation, the proposed scheme utilizes the Canny edge detector on pupil-detected eye-images. It then uses OMT by setting $R_{m}$ to 40 pixels and $R_{M}$ to 80 pixels to find the rough inner iris-boundary.
2.1.3. Precise Inner Iris-Boundary Locating with Delogne-Kåsa Circle Fitting. The points detected by OMT are tabulated in the form of ordered pairs $\left(x_{1}, y_{1}\right),\left(x_{2}, y_{2}\right), \ldots,\left(x_{n}, y_{n}\right)$. For determining a more accurate iris-boundary, a robust technique for efficiently fitting circles through noisy data, the Delogne-Kåsa circle fitting scheme, is used to locate the irisboundaries. It is illustrated as follows.

In Chan's circle functional model [11], points $\left(x_{i}, y_{i}\right), i=$ $1,2, \ldots, n$, which are taken from the circumference of a circle, are formed as

$$
\begin{gathered}
x_{i}=a+\rho \cos \varphi_{i}+\xi_{i}, \\
y_{i}=b+\rho \sin \varphi_{i}+\eta_{i}, \quad i=1,2, \ldots, n
\end{gathered}
$$

where $(a, b)$ is the center of the best circle, $\rho$ is its radius, $\varphi_{i}$ is the phase of the $i$ th pair of measurements, and $\xi_{i}$ and $\eta_{i}$ represent the measurement errors that are independent and identically distributed normal random variables with zero means and standard deviations $\sigma_{\xi}$ and $\sigma_{\eta}$, respectively. The best circle fitting is to find a circle whose circumference is as close as possible to the measured data $\left(x_{i}, y_{i}\right), i=1,2, \ldots n$.

Instead of the maximum-likelihood estimator scheme used in [11], the Delogne-Kåsa estimates scheme [12] searches the most suitable parameters $a, b$, and $\rho$ so as to minimize the cost function:

$$
E=\sum_{i=1}^{n}\left\{\left(x_{i}-a\right)^{2}+\left(y_{i}-b\right)^{2}-\rho^{2}\right\}^{2} .
$$



be

By changing parameter $c=a^{2}+b^{2}-\rho^{2}$, it is reduced to

$$
(\widehat{a}, \widehat{b}, \widehat{c})=\arg \min _{(a, b, c)} \sum_{i=1}^{n}\left\{x_{i}^{2}+y_{i}^{2}-2 a x_{i}-2 b y_{i}+c\right\}^{2} .
$$

By taking partial derivatives with respect to $a, b$, and $c$, respectively, the cost function is minimized when

$$
\widehat{c}=\frac{1}{n} \sum_{i=1}^{n}\left\{x_{i}^{2}+y_{i}^{2}-2 a x_{i}-2 b y_{i}\right\} .
$$

The result,

$$
(\widehat{a}, \widehat{b})=\arg \min _{(a, b)} \sum_{i=1}^{n}\left\{x_{i}^{2}+y_{i}^{2}-2 a x_{i}-2 b y_{i}+\widehat{c}\right\}^{2},
$$

can be proved to have the solution

$$
(\widehat{a}, \widehat{b})^{T}=A^{\#}(R(\vec{u}+\vec{v}))=(R(S+T))^{\#}(R(\vec{u}+\vec{v})),
$$

where $A=R(S+T), A^{\#}=\left(A^{T} A\right)^{-1} A^{T}$, and the superscript \# indicates the Moore-Penrose generalized inverse:

$$
\begin{gathered}
S=\left(\begin{array}{cc}
a+\rho \cos \phi_{1} & b+\rho \sin \phi_{1} \\
\vdots & \vdots \\
a+\rho \cos \phi_{n} & b+\rho \sin \phi_{n}
\end{array}\right)_{n \times 2}, \\
T=\left(\begin{array}{cc}
\xi_{1} & \eta_{1} \\
\vdots & \vdots \\
\xi_{n} & \eta_{n}
\end{array}\right)_{n \times 2}, \\
\vec{u}=\left(\left(a+\rho \cos \phi_{1}\right)^{2}+\left(b+\rho \sin \phi_{1}\right)^{2}\right) \\
\left.\ldots,\left(\left(a+\rho \cos \phi_{n}\right)^{2}+\left(b+\rho \sin \phi_{n}\right)^{2}\right)\right)_{1 \times n}^{T} \\
\vec{v}=\left(\left(2\left(a+\rho \cos \phi_{1}\right) \xi_{1}+2\left(b+\rho \sin \phi_{1}\right) \eta_{1}+\xi_{1}^{2}+\eta_{1}^{2}\right)\right. \\
\left.\ldots,\left(2\left(a+\rho \cos \phi_{n}\right) \xi_{n}+2\left(b+\rho \sin \phi_{n}\right) \eta_{n}+\xi_{n}^{2}+\eta_{n}^{2}\right)\right)_{1 \times n}^{T} \\
R=I_{n \times n}-\frac{Q_{n \times n}}{n},
\end{gathered}
$$

where $I_{n \times n}$ is the $n \times n$ identity matrix and $Q_{n \times n}$ is the $n \times n$ matrix with all elements being 1 .

The proposed iris-boundary locating procedure using $\mathrm{DKCF}$ is given as follows.

Step 1. Set the points detected by OMT as inliers.

Step 2. Fit a circle $C$ by applying DKCF to the inliers obtained in Step 1.

Step 3. For each point $p\left(x_{i}, y_{i}\right)$ of inliers, evaluate the Mahalanobis distance $\mathrm{MD}_{i}$ with

$$
\mathrm{MD}_{i}=\sqrt{\left[x_{i}-\mu_{x}, y_{i}-\mu_{y}\right] V^{-1}\left[x_{i}-\mu_{x}, y_{i}-\mu_{y}\right]^{T}},
$$

where $V$ is $2 \times 2$ the covariance matrix, $\mu_{x}$ is the mean of $x$ component, and $\mu_{y}$ is the mean of $y$-component of inliers.
Step 4. Find the mean $\mu_{\mathrm{MD}}$ and the standard deviation $\sigma_{\mathrm{MD}}$ of the Mahalanobis distance distribution of inliers and test all the points in inliers. If a point's Mahalanobis distance less than $\mu_{\mathrm{MD}}+1.5 \sigma_{\mathrm{MD}}$, then reclassify it as an inlier; otherwise, reclassify it as an outlier.

Step 5. Refit circle C with Delogne-Kåsa circle fitting scheme for the updated inliers.

Step 6. Repeat Steps 3, 4, and 5 until the fitting circle does not change any further.

Once the pupil region is detected, it will be removed from the eye-image. The outer iris-boundary is a near circular boundary between the iris and sclera. In a pupil-removed grayscale eye-image, the darker regions consist of the iris and eyelashes, and the brighter regions consist of the sclera and eyelids. Hence Otsu's binarization scheme is applied on the pupil-removed eye-image to obtain the candidate iris areas.

2.1.4. Binarization Using Otsu's Algorithm. The Otsu thresholding scheme, proposed by Otsu [13], first normalizes the histogram of pixels of the pupil-removed eye-image as a probability distribution and divides all pixels into two classes by a threshold. The occurrence probabilities, the means, and the variances of each class are then evaluated. And, the within-class variance, the between-class variance, and the total variance are also evaluated. The optimal threshold is evaluated using the discriminated criterion which maximizes the between-class variance and minimizes the within-class variance at the same time. In general the biggest black region in a binary eye-image obtained by the Otsu filter will contain the iris region and other smaller black regions are noises. The proposed algorithm labels these black regions and evaluates their size to extract the biggest black region as the candidate iris region.

2.1.5. Precise Outer Iris-Boundary Location. The Sobel edge filter is applied on the candidate iris region to extract an initial outer iris-boundary. Then the proposed iris-boundary locating procedure using DKCF is applied to extract a more precise outer iris-boundary by setting the inner radius $R_{m}$ to 170 pixels and the outer radius $R_{M}$ to 200 pixels in the OMT step. Figure 3 shows the result of each step in the proposed inner and outer boundaries localization algorithm, respectively.

2.2. Stage 2: Eyelid Locating. For a noisy iris image, the iris's upper region is frequently occluded by the upper eyelid and eyelashes either partly or completely. The lower region is usually partly occluded by the lower eyelid. Noisy iris images can cause incorrect matching and adversely affect the iris recognition rate. In order to extract the iris accurately and effectively, eyelids and eyelashes should be filtered out at high precision.

The common methods to filter out the eyelid are as follows: (i) utilize an edge detector to detect the edges of an eye-image and then apply a linear Hough transform on the eye-image edge map to locate the eyelid boundaries 


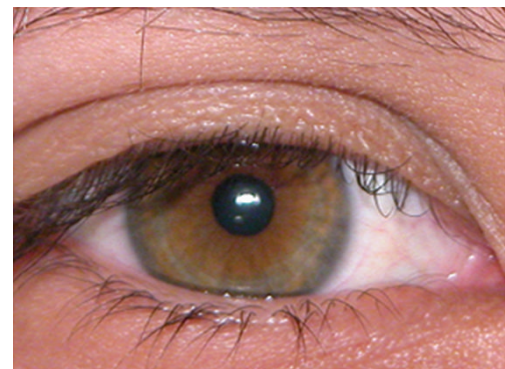

(a)

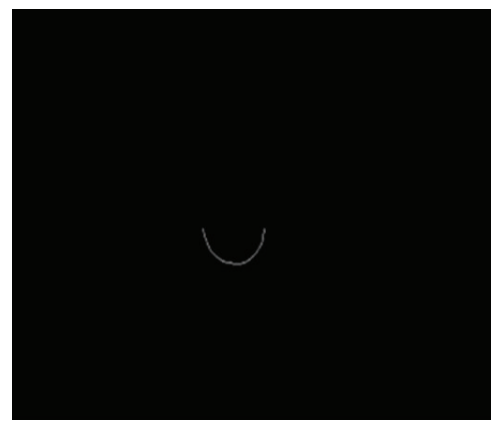

(d)

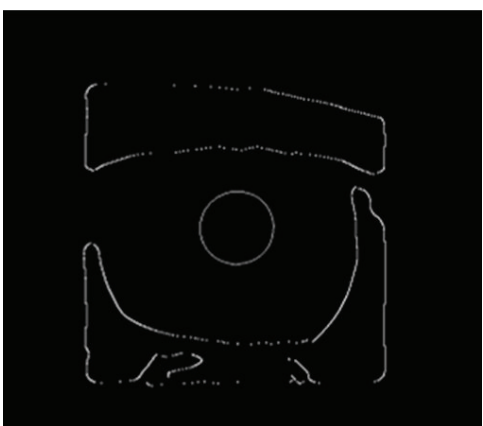

(g)

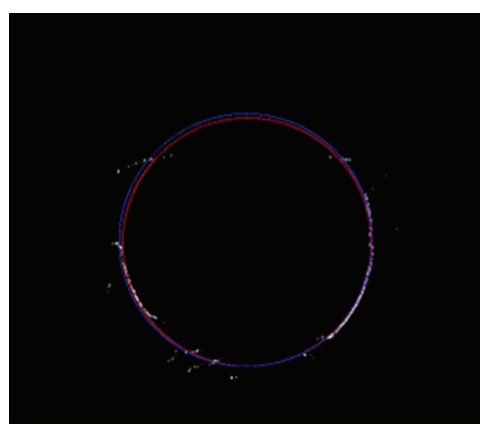

(j)

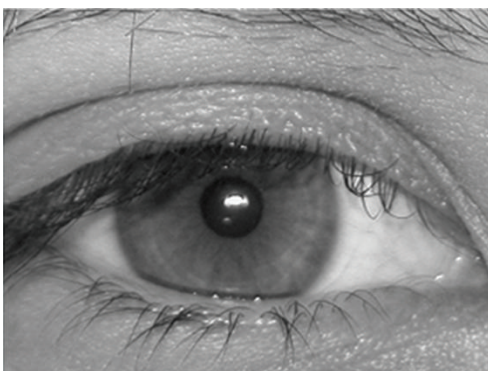

(b)

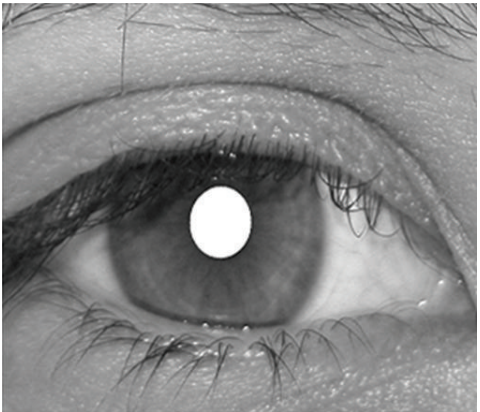

(e)

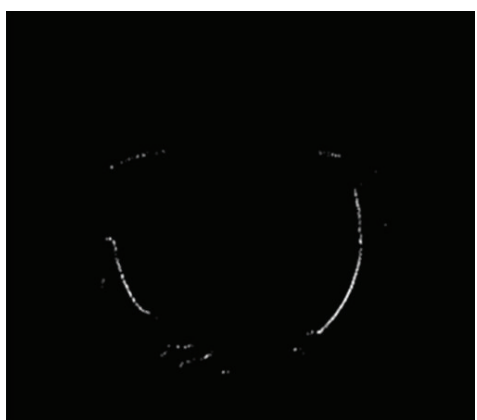

(h)

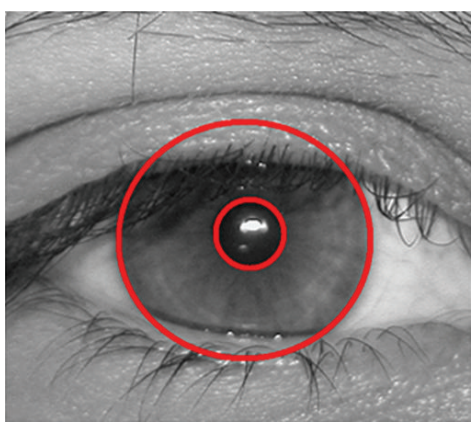

(k)

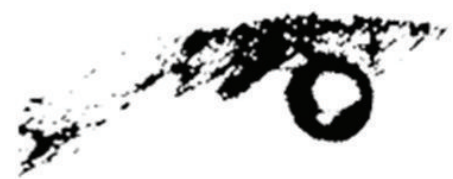

(c)

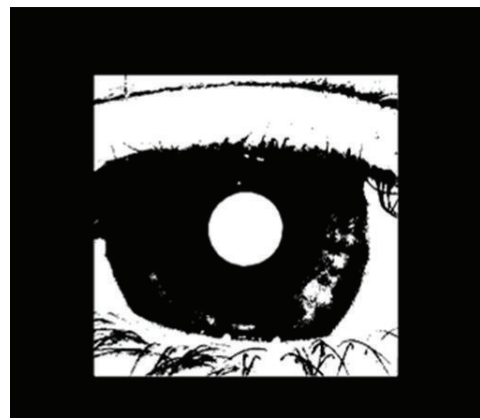

(f)

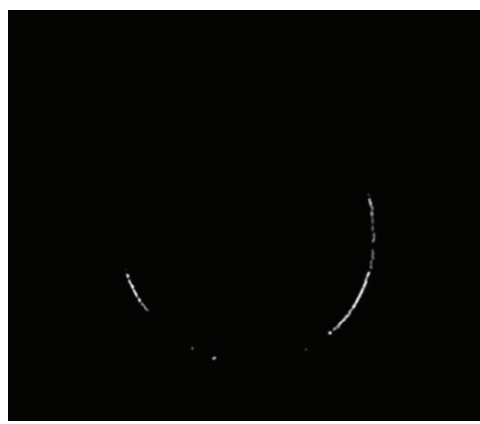

(i)

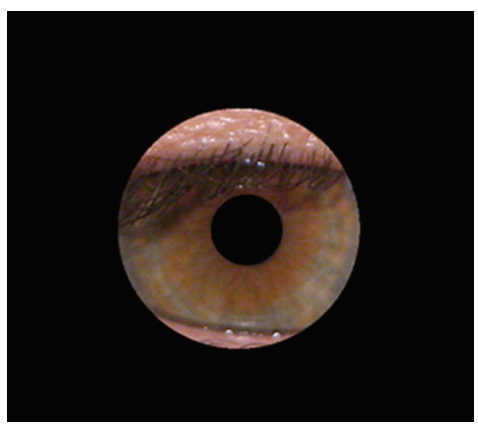

(1)

FIGURE 3: The result of the proposed inner and outer boundaries localization algorithm: (a) original RGB color iris image, (b) corresponding $y$-component image, (c) candidate pupil region after outliers detection and morphological opening operation, (d) detected inner iris-border, (e) pupil-removed eye-image in grayscale, (f) candidate iris region after redundant areas removal, (g) edge map of (f) created by Sobel detector, (h) outer iris-border by OMT from the edge map, (i) outer iris-border after DKCF three times, (j) the best fit circle for final inliers (circle in red) and the best fit circle for points detected by OMT (circle in blue), (k) inner and outer iris-boundaries located by the proposed scheme, and (l) the detected iris region. 


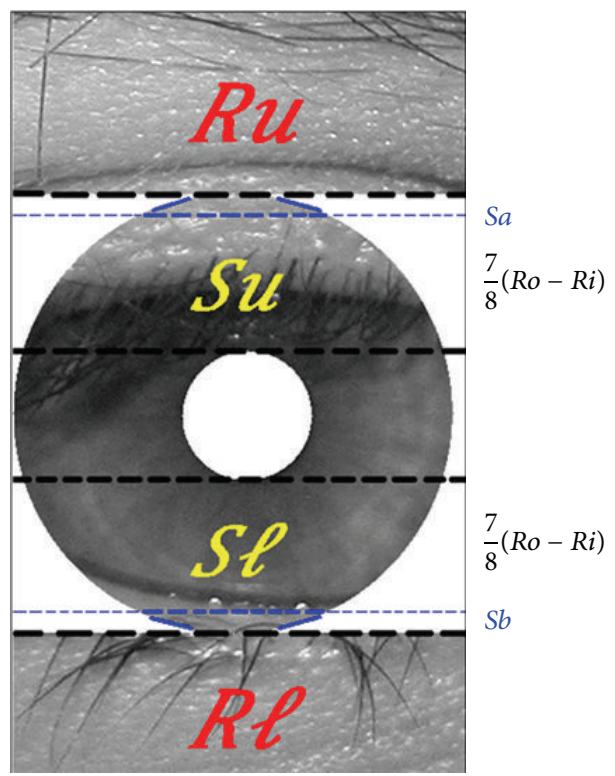

(a)

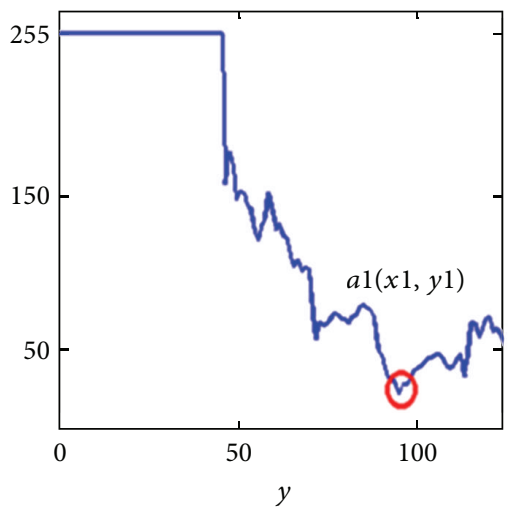

(b1)

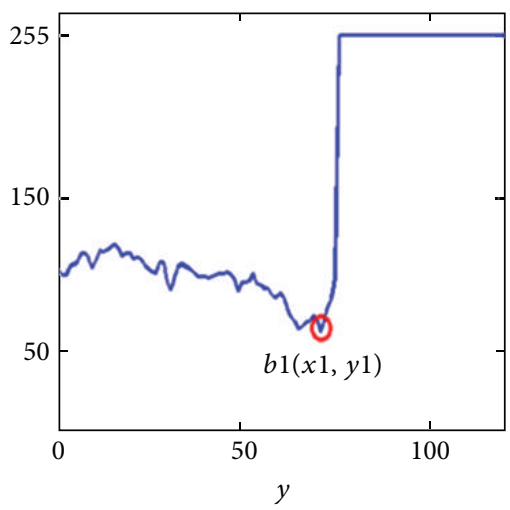

(c1)

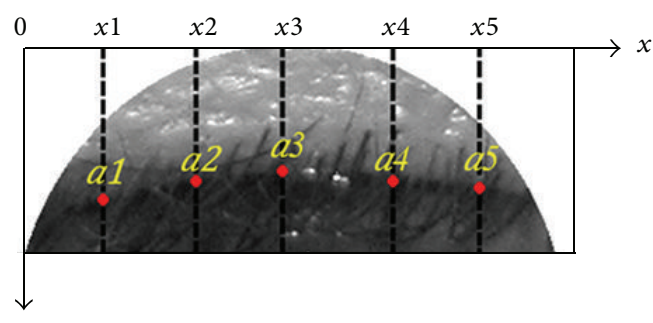

(b)

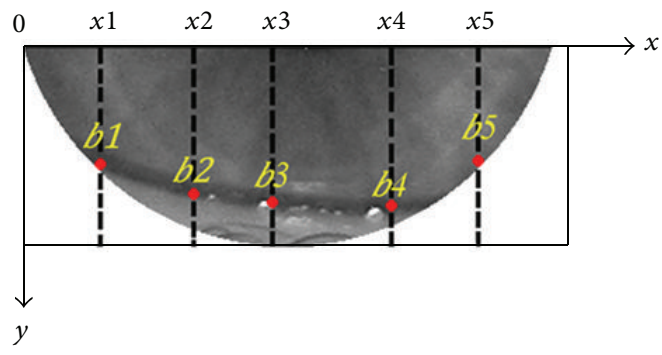

(c)

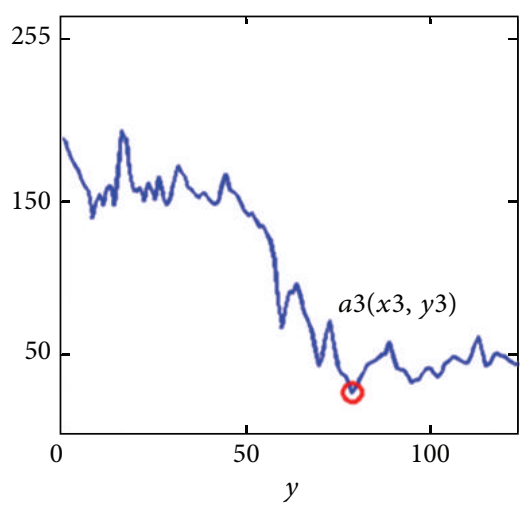

(b3)

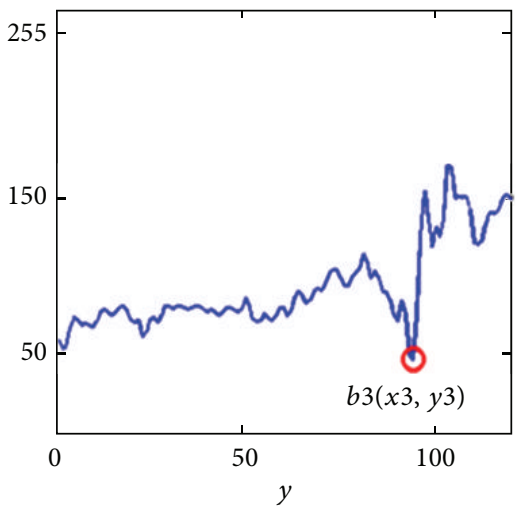

(c3)

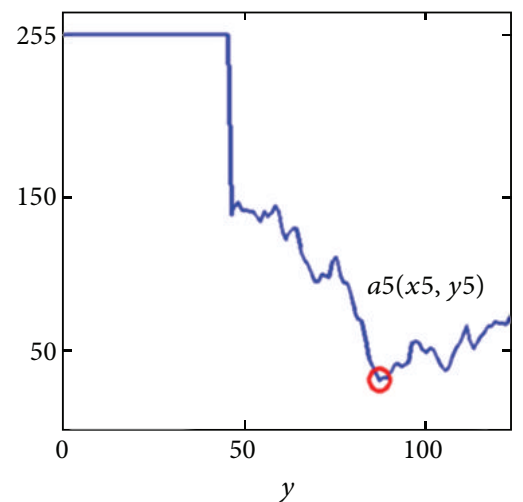

(b5)

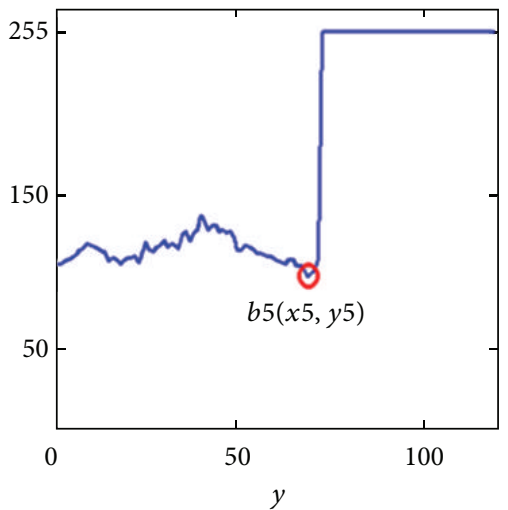

(c5)

FIGURE 4: Segmented regions and columns' pixel value distributions with respect to $y$-coordinate.

and mask out the upper and lower eyelids. These linear approximations are simple but rough [14]. (ii) Extract the boundary information of an eye-image and then perform the curve integral-differential operator on the eye-image edge map to locate the eyelid. These linear approximations can be significantly affected by the doublefold and eyelash roots [14]. In addition, other edge points caused by eyelashes and the texture of the iris patterns may appear in the edge map. 
TABLE 1: Performances of the proposed algorithm for eyelash occlusion eye-images.

\begin{tabular}{|c|c|c|c|c|c|}
\hline Image & Stages & $\mathrm{ME}$ & RFAE & ACC & EMM \\
\hline \multirow{3}{*}{ Img_5_4 } & Stage_1 & 0.0503 & 0.1508 & 0.9497 & 0.9800 \\
\hline & Stage_2 & 0.0363 & 0.0574 & 0.9637 & 0.9905 \\
\hline & Stage_3 & 0.0295 & 0.0122 & 0.9705 & 0.5732 \\
\hline \multirow{3}{*}{ Img_10_1 } & Stage_1 & 0.0349 & 0.1097 & 0.9651 & 0.8689 \\
\hline & Stage_2 & 0.0296 & 0.0626 & 0.9704 & 0.8149 \\
\hline & Stage_3 & 0.0220 & 0.0106 & 0.9780 & 0.8990 \\
\hline \multirow{3}{*}{ Img_33_1 } & Stage_1 & 0.0517 & 0.2819 & 0.9483 & 0.8316 \\
\hline & Stage_2 & 0.0350 & 0.2066 & 0.9650 & 0.9547 \\
\hline & Stage_3 & 0.0194 & 0.1130 & 0.9806 & 0.9298 \\
\hline \multirow{3}{*}{ Img_43_1 } & Stage_1 & 0.1003 & 0.4387 & 0.8997 & 0.9712 \\
\hline & Stage_2 & 0.0550 & 0.2638 & 0.9450 & 0.9921 \\
\hline & Stage_3 & 0.0357 & 0.1450 & 0.9643 & 0.9821 \\
\hline \multirow{3}{*}{ Img_46_3 } & Stage_1 & 0.0839 & 0.4058 & 0.9161 & 0.9491 \\
\hline & Stage_2 & 0.0437 & 0.2221 & 0.9563 & 0.9582 \\
\hline & Stage_3 & 0.0316 & 0.1169 & 0.9684 & 0.6572 \\
\hline \multirow{3}{*}{ Img_74_3 } & Stage_1 & 0.0303 & 0.1218 & 0.9697 & 0.8565 \\
\hline & Stage_2 & 0.0294 & 0.1173 & 0.9706 & 0.8651 \\
\hline & Stage_3 & 0.0229 & 0.0668 & 0.9771 & 0.4453 \\
\hline
\end{tabular}

TABLE 2: MOEs of the proposed algorithm for the UBIRIS.vl.

\begin{tabular}{|c|c|c|c|c|c|}
\hline Image & Stages & $\mathrm{ME}$ & RFAE & ACC & EMM \\
\hline \multirow{3}{*}{ Maximum } & Stage_1 & 0.1048 & 0.5172 & 0.9967 & 0.9830 \\
\hline & Stage_2 & 0.0550 & 0.4338 & 0.9967 & 0.9830 \\
\hline & Stage_3 & 0.0448 & 0.4187 & 0.9967 & 0.9830 \\
\hline \multirow{3}{*}{ Minimum } & Stage_1 & 0.0033 & 0.0005 & 0.8952 & 0.1409 \\
\hline & Stage_2 & 0.0033 & 0.0005 & 0.9450 & 0.1041 \\
\hline & Stage_3 & 0.0033 & 0.0005 & 0.9552 & 0.1041 \\
\hline \multirow{3}{*}{ Mean } & Stage_1 & 0.0372 & 0.1406 & 0.9628 & 0.8101 \\
\hline & Stage_2 & 0.0226 & 0.0857 & 0.9774 & 0.7031 \\
\hline & Stage 3 & 0.0187 & 0.0594 & 0.9813 & 0.6704 \\
\hline \multirow{3}{*}{ Standard } & Stage_1 & 0.0212 & 0.1183 & 0.0212 & 0.1260 \\
\hline & Stage_2 & 0.0123 & 0.0683 & 0.0123 & 0.2500 \\
\hline & Stage_3 & 0.0087 & 0.0445 & 0.0087 & 0.2370 \\
\hline
\end{tabular}

The shape of a real eyelid boundary is always not a smooth curve (or a smooth line segment). The above-mentioned schemes mentioned yield less precise eyelid detection.

To overcome the problems occurring in these schemes, an improved strategy for eyelid detection is proposed. Because eyelids occlude areas outside iris-boundaries which are not relevant, the proposed eyelid detection approach is performed only inside the iris region to increase the detection speed. After the inner and outer boundaries are located, the grayscale eye-image is segmented into three areas by two vertical lines tangent at the left and right most points in the outer boundary. The middle area is taken as the residual image and divided into seven nonoverlapped regions using six horizontal line segments: $R u, S a, S u, S l, S b$, and $R l$ as shown in Figure 4.

For detecting the upper eyelid which occludes the iris, the average grey values of a rectangular patch $R u$ just above the top of the iris' outer boundary and the segment $S a$ just below the top of the iris' outer boundary are evaluated first. The iris is treated as occluded by the upper eyelid when the absolute difference between the two average grey values is less than 10 . The eyelid boundary usually appears darker than the skin and is almost always adjacent to the iris. Figures $4(\mathrm{~b} 1)$, 4(b3), 4(b5), 4(c1), 4(c3), and 4(c5) show the column pixel value distributions with respect to the $y$-coordinate in the upper and lower segments. Once an iris region is detected as occluded by the upper eyelid, the proposed algorithm detects the darkest pixel of each column in the upper part of the iris region as the eyelid boundary point in that column. The pixels above the eyelid boundary are removed for each column in the union regions of $\mathrm{Sa}$ and $\mathrm{Su}$. Lower eyelid removal that occludes the iris is similar to the upper eyelid removal. Figure 5 shows the result of each step in the proposed eyelids localization algorithm for an eye-image occluded by eyelids. 


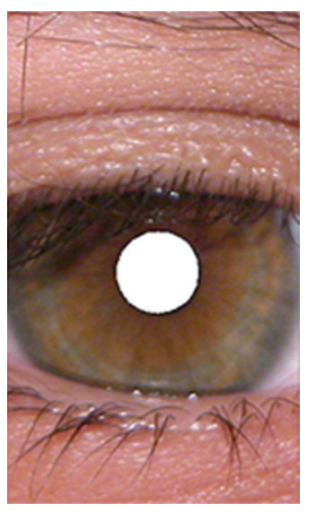

(a)

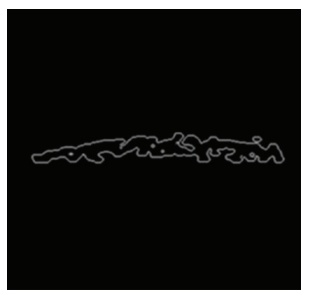

(f)

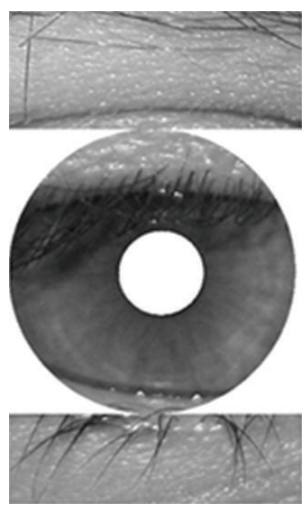

(b)

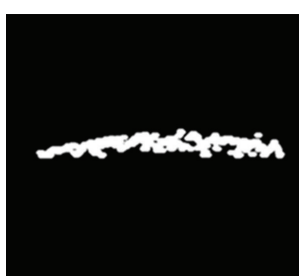

(g)

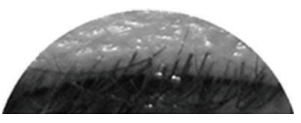

(c)

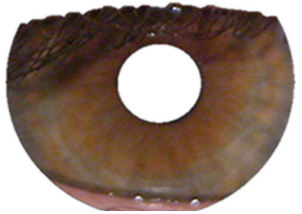

(h)

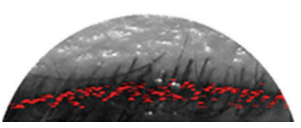

(d)

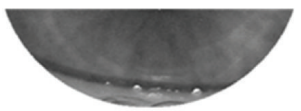

(i)

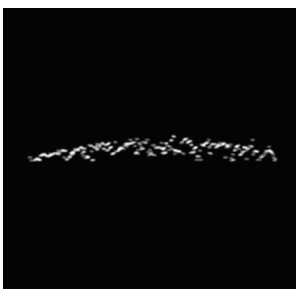

(e)

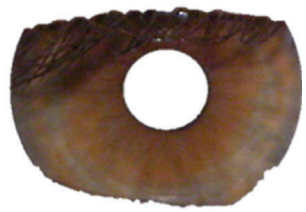

(j)

FIGURE 5: The result of the proposed eyelid segmentation algorithm: (a) middle area of an eye-image occluded by eyelids, (b) grayscale image of (a) after inner and outer boundaries localization, (c) upper segment of the disk region of iris, (d) upper eyelid boundary detected by the proposed scheme, (e) upper eyelid boundary extracted by the proposed scheme, (f) upper eyelid boundary after morphological dilation operation, (g) edge map of (f), (h) iris after upper eyelid segmented, (i) lower segment of the disk region of iris, and (j) iris after upper and lower eyelids segmented.

2.3. Stage 3: Eyelash and Shadow Detection. Eyelashes and shadows are other occlusions that degrade iris segmentation. Detecting eyelashes and shadows requires the choice of proper features and a classification procedure due to their complexity and randomness. There are three schemes for detecting eyelashes and shadows in the public literature: (i) Kong and Zhang [15] classified eyelashes into multiple eyelashes (or shadows) and separable eyelashes. Multiple eyelashes were detected using the variance in intensity and separable eyelashes were detected using the one-dimensional Gabor filter. This method is time-consuming because of global region searching. (ii) Huang et al. [16] detected eyelashes using a bank of Log-Gabor filters to extract the edge information based on phase congruency. This method may recognize the area between two adjacent eyelashes as an eyelash region. (iii) Yuan and He [17] detected eyelashes using the mean and standard deviation of an eyelash template manually taken from a test image. This method is affected significantly by the illumination variation and the template.

Based on the fact that eyelashes always appear under the upper eyelid and the eyelash region is darker than the iris region, the proposed algorithm utilizes the statistical iris region characteristic intensity to detect eyelashes within the iris area. The eyelid-removed iris image is divided into three nonoverlapped regions with equal height: the region just below the upper eyelid, the region just above the lower eyelid, and the middle region using two horizontal line segments. The algorithm evaluates the mean $\mu_{u}$ of intensity in the region just below upper eyelid and evaluates the mean $\mu_{l}$ and standard deviation $\sigma_{l}$ of intensity in the region just above lower eyelid, respectively. If the relative difference ratio from $\mu_{u}$ to $\mu_{l}$ is larger than a threshold $T_{u}=0.1$, then the eyelash is detected. Here, the relative difference is defined by the following equation:

$$
\operatorname{RDR}=\frac{\left(\mu_{l}-\mu_{u}\right)}{\mu_{l}} .
$$

If the intensities of a pixel and more than one of its 8neighbor are less than $\mu_{l}-2.5 \sigma_{l}$ at the same time, then this pixel is taken as an eyelash pixel and removed. Figure 6 shows the proceeding result of the proposed eyelash segmentation algorithm.

\section{Experimental Result}

The proposed iris region extraction scheme was applied to the iris image database, UBIRIS.v1, which has 1214 eye-images collected from 241 eyes and the size of each eye-image is $600 * 800 * 3$ pixels with a bit-depth of 8 bits. Each of these 1214 test images was labelled by an expert to carefully construct the ground-truth segmentation for evaluating the proposed algorithm's segmentations.

In this paper, several measures of errors (MOEs) are conducted to measure the performance of the proposed algorithm. They are misclassification error (ME), accuracy (ACC), relative foreground area error (RFAE), and edge mismatch (EMM) [18]. ME, RFAE, and EMM vary from 0 for 


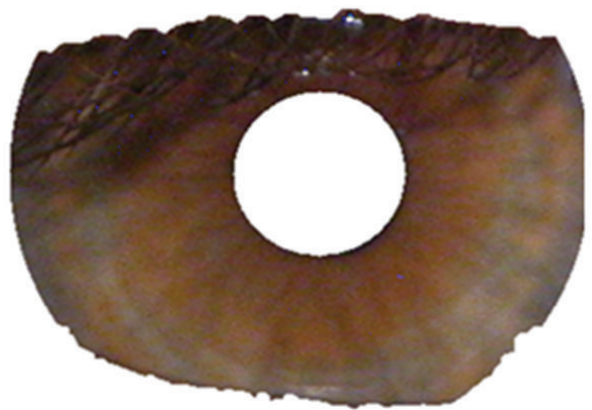

(a)

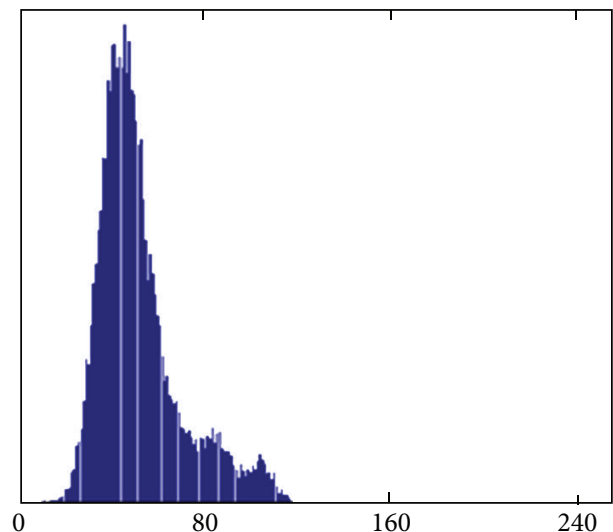

(c)

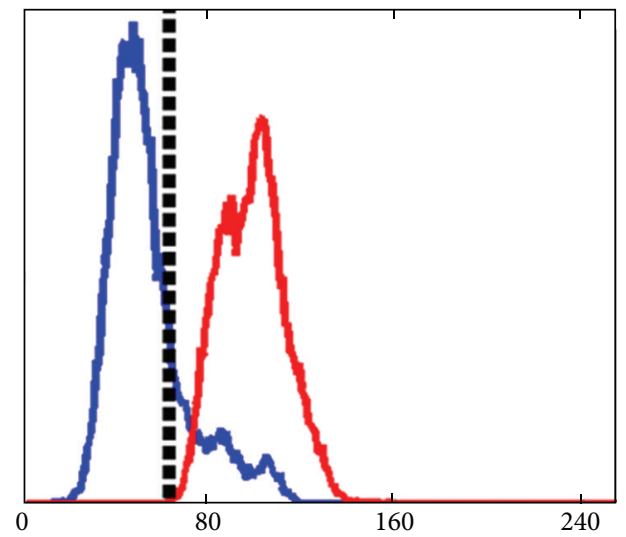

(e)

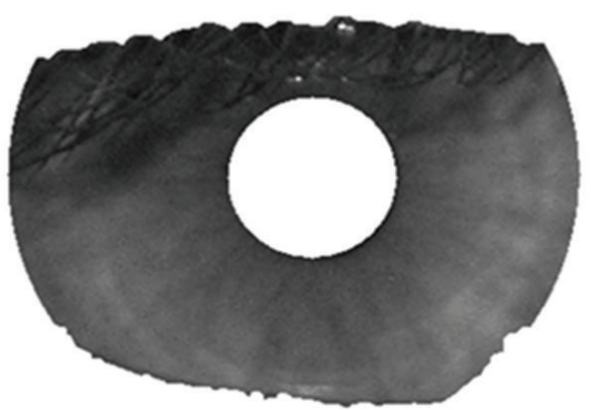

(b)

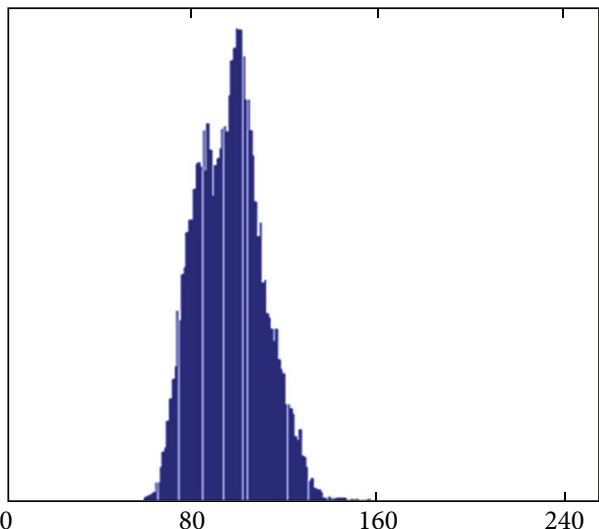

(d)

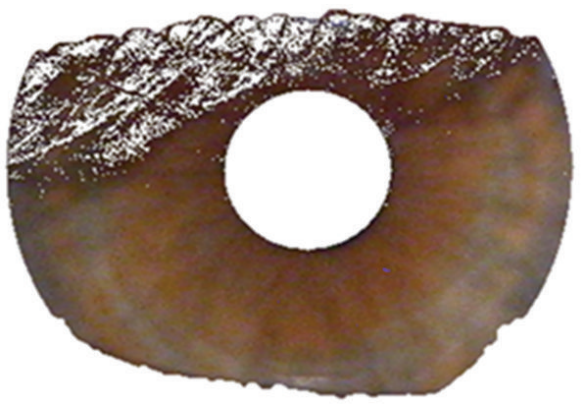

(f)

Figure 6: The result of the proposed eyelash segmentation algorithm: (a) iris image with eyelids removal, (b) grayscale image of (a), (c) the histogram of the region just below upper eyelid, (d) the histogram of the region just above lower eyelid, (e) the histogram-plot of the below upper eyelid and the above lower eyelid regions, where the black vertical dash line indicates the intensity threshold for eyelashes detection, and (f) the result of the proposed eyelash segmentation algorithm.

TABLE 3: The accuracy of relative methods for the 1214 eye-images of the UBIRIS.v1.

\begin{tabular}{lccc}
\hline Method & Accuracy (\%) & RFAE & Time cost (sec.) \\
\hline Camus and Wildes [5] & 96.78 & 0.0452 & 3.12 \\
Proença and Alexandre [6] & 98.02 & 0.0446 & 2.3 \\
de Martin-Roche et al. [4] & 77.18 & 0.0566 & 2.91 \\
Proposed & 98.13 & 0.0445 & 2.78 \\
\hline
\end{tabular}


a perfectly correct segmentation to 1 for a completely error case. These performance measures are illustrated as follows:

$$
\begin{aligned}
\mathrm{ME} & =\frac{(\mathrm{FN}+\mathrm{FP})}{(\mathrm{TP}+\mathrm{FN}+\mathrm{TN}+\mathrm{TP})}, \\
\mathrm{ACC} & =\frac{(\mathrm{TP}+\mathrm{TN})}{(\mathrm{TP}+\mathrm{TN}+\mathrm{FN}+\mathrm{FP})},
\end{aligned}
$$

RFAE

$$
= \begin{cases}\frac{(\mathrm{TP}+\mathrm{FN})-(\mathrm{FP}+\mathrm{TP})}{\mathrm{TP}+\mathrm{FN}} & \text { if }(\mathrm{FP}+\mathrm{TP})<(\mathrm{TP}+\mathrm{FN}) \\ \frac{(\mathrm{FP}+\mathrm{TP})-(\mathrm{TP}+\mathrm{FN})}{\mathrm{FP}+\mathrm{TP}} & \text { if }(\mathrm{FP}+\mathrm{TP}) \geq(\mathrm{TP}+\mathrm{FN}),\end{cases}
$$

where TP, TN, FP, and FN represent the areas of true positive, true negative, false positive, and false negative, respectively. Thus,

$$
\mathrm{EMM}=\frac{1-n_{\mathrm{C}}}{\left(n_{\mathrm{C}}+\alpha \sum_{k \in E_{\mathrm{gm}}} \delta(k)+\beta \sum_{k \in E_{\mathrm{ee}}} \delta(k)\right)},
$$

where $n_{c}$ indicates the number of intersection edge pixels found between the extracted objects and the ground-truth object, $E_{\mathrm{gm}}$ is the set of edge pixels of the ground-truth objects that are missed in the extracted objects, and $E_{\mathrm{ee}}$ is the set of excess extracted edge pixels of the extracted objects that are not edge pixels of the ground-truth objects. $\alpha$ and $\beta$ are two penalties associated with pixels that belong to $E_{\mathrm{gm}}$ and $E_{\mathrm{ee}}$, respectively. $\delta(k)$ indicates a distance function defined as

$$
\delta(k)= \begin{cases}d_{k}, & \text { if } \delta(k)<d_{m} \\ D_{\max }, & \text { otherwise, }\end{cases}
$$

where $d_{k}$ is the Euclidean distance between the $k$ th excess edge pixel and a complementary edge pixel within a search area determined by the parameter $d_{m}$ and $D_{\max }$ is a designated constant.

Table 4 shows some visual results of the proposed iris segmentation algorithm on eyelids and eyelash occlusions iris images from the UBIRIS.v1 database. Row 2 shows the original eye-images, row 3 shows the corresponding ground-truth iris images labelled by an expert radiologist, and row 4 shows the segmented results of the presented algorithm. Table 1 shows the corresponding MOEs of Table 4. To illustrate the performance of each stage of the proposed iris segmentation algorithm, the MOE statistical analysis of each stage on whole eye-images in the UBIRIS.v1 database is shown in Table 2 . As seen in Table 2 the segmentation results were improved stage by stage. In the accuracy, the average is 0.9628 and the minimum is 0.8952 in Stage 1 , the average is 0.9774 and the minimum is 0.9450 in Stage 2, and the average is 0.9813 and the minimum is 0.9552 in Stage 3. In the RFAE, the average is 0.1406 and the maximum is 0.5172 in Stage 1, the average is 0.0857 and the maximum is 0.4338 in Stage 2, and the average is 0.0594 and the maximum is 0.4187 in Stage 3.

The successful segmentation rate for each stage with respect to the false positive rate, the $\mathrm{ME}$, and the RFAE are evaluated and plotted in Figure 7. The successful segmentation rate is defined as the ratio of the number of eyeimages with segment results achieving a threshold to the size of the eye-image database. There are $67 \%, 92 \%$, and $98 \%$ database images, where the false positive rate was no greater than 0.01 after Stage 1, Stage 2, and Stage 3, respectively. There are $66 \%, 88 \%$, and $99 \%$ images of database whose ME is no more than 0.01 after Stage 1, Stage 2, and Stage 3, respectively. There are $73 \%, 90 \%$, and $98 \%$ images of database whose RFAE is no more than 0.25 after Stage 1 , Stage 2, and Stage 3, respectively. The experimental results show that the proposed iris segmentation algorithm is of high performance and robust even works on the noisy eye-images.

The proposed algorithm, Camus and Wildes' method [5], Proenca and Alexandre's method [6], and MartinRoche et al.s method [4] are compared. The average accuracy of these methods on the 1214 eye-images in the UBIRIS.v1 database is given in Table 3 . Table 3 shows that the means of accuracy and RFAE of the three related methods are larger than those of the proposed scheme. Camus and Wildes' method is accurate on good quality and noise-free iris images. The proposed method takes an additional step, the DelogneKåsa circle fitting step, to process the circles extracted by the Circular Hough transform. Compared to Proenca and Alexandre's method, the time cost is increased a little and the accuracy is higher in the proposed method. Table 3 shows that the proposed scheme gives precise extractions for irises. Moreover, Table 5 is given to compare results obtained by using the proposed method and the method of Vatsa et al. [19] on six heavy noise iris images. Vatsa et al's method fails to perform on the six heavy noise iris images, but the proposed scheme can perform with an average accuracy of $98.82 \%$.

\section{Conclusions}

Compared to the other physiological characteristics, iris recognition systems are considered as the most valuable and reliable biometric recognition systems in the world. Moreover, automatically and reliably segmenting the iris from the captured eye-images is considered as the most important stage in an iris recognition system, which greatly influences the overall recognition accuracy and processing speed of the whole system. Iris segmentation is to extract the iris from its surrounding noises, such as pupil, sclera, eyelashes, and eyebrows. A novel iris segmentation scheme is proposed to remove as much noise as possible. Based on edge maps the presented algorithm uses the orientation matching transform to find the rough outer and inner irisboundaries. Then a mathematical technique, Delogne-Kåsa circle fitting, is employed to eliminate the outlier points of the rough outer and inner iris-boundaries to extract a more precise iris area from an eye-image. In the extracted iris region, the proposed algorithm utilizes the differences among the intensity and position characteristics of the iris, eyelid, and eyelashes to detect these noises to obtain highly accurate iris segmentation. For evaluating the performance of the proposed iris segmentation algorithm, the algorithm is implemented on a personal computer with an Intel Pentium $2.83 \mathrm{GHz}$ Core (TM) 2 Duo CPU running window XP and 


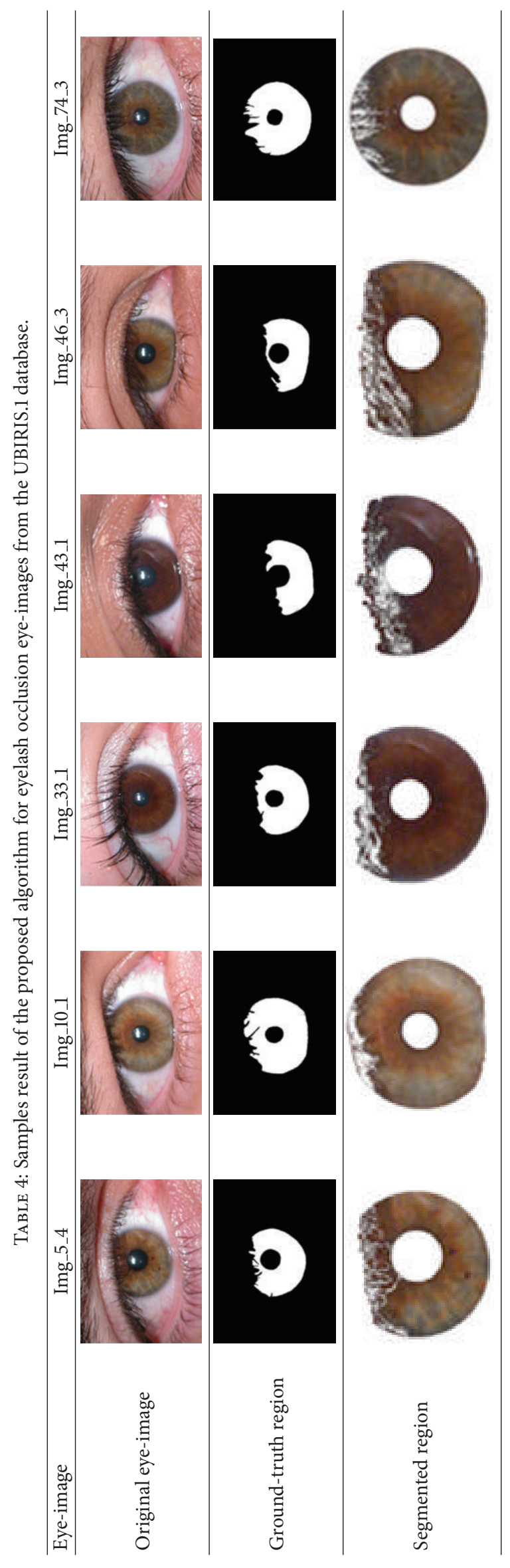




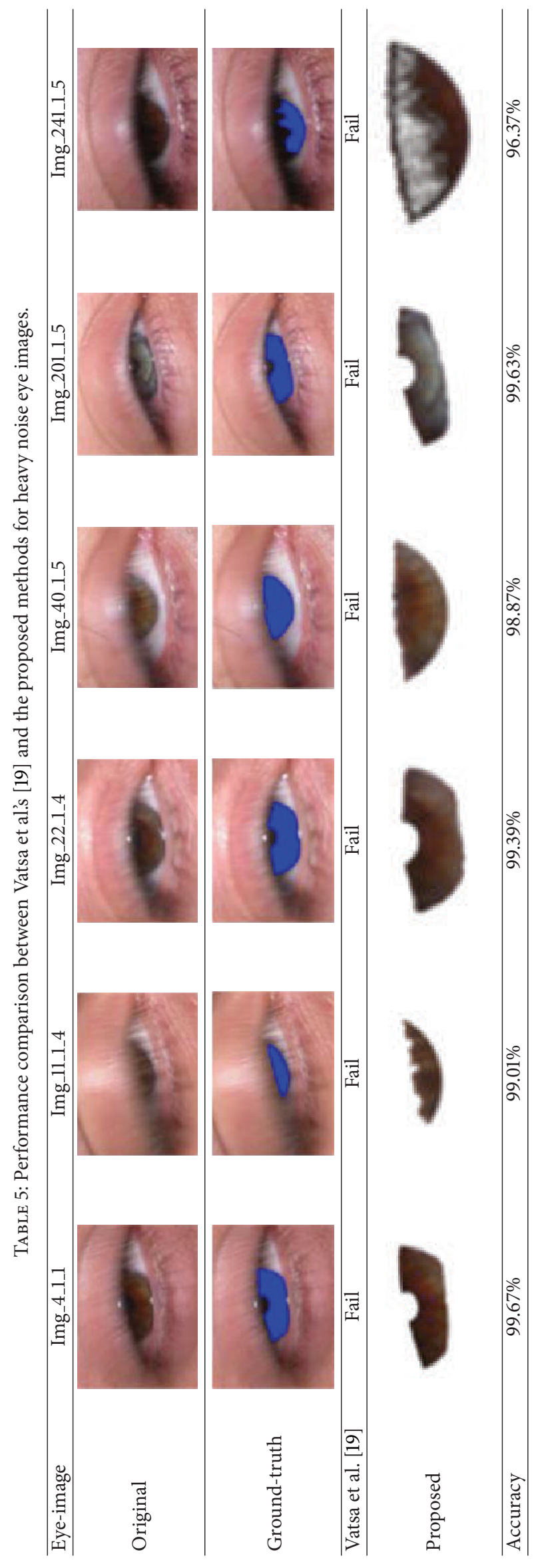




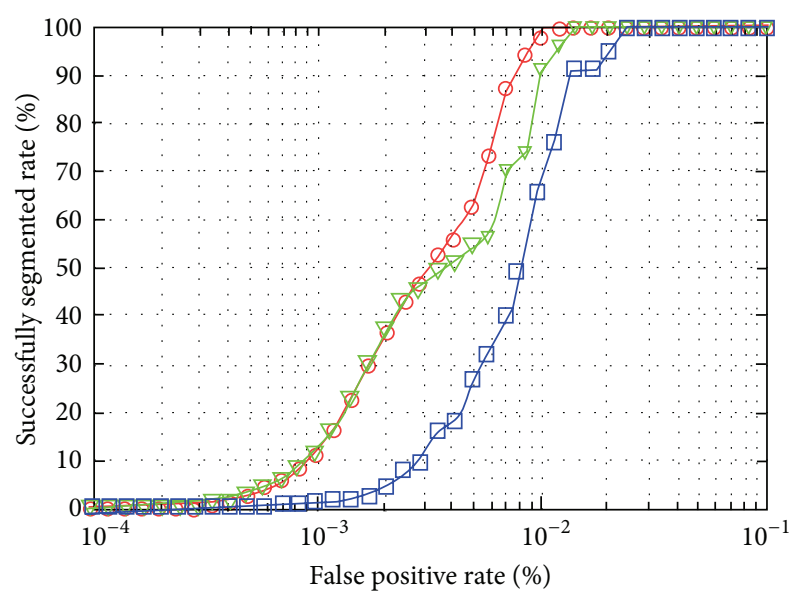

(a)

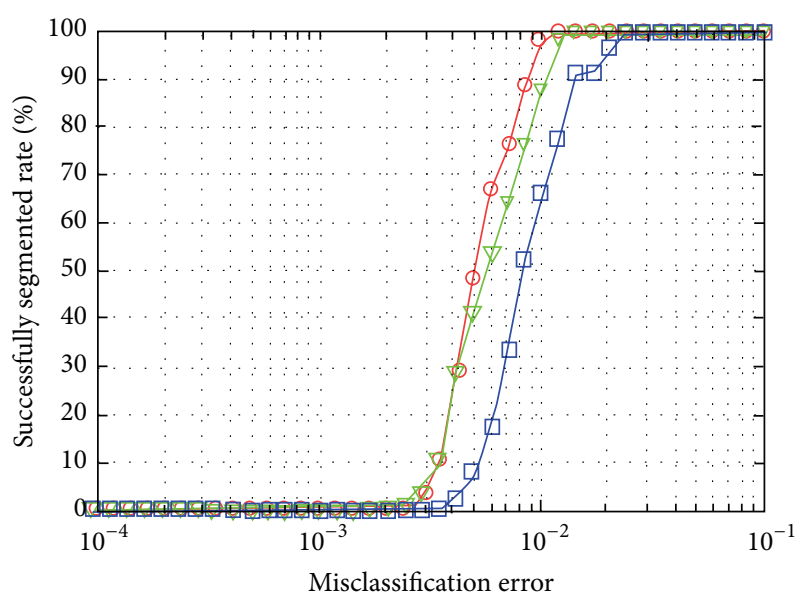

(b)

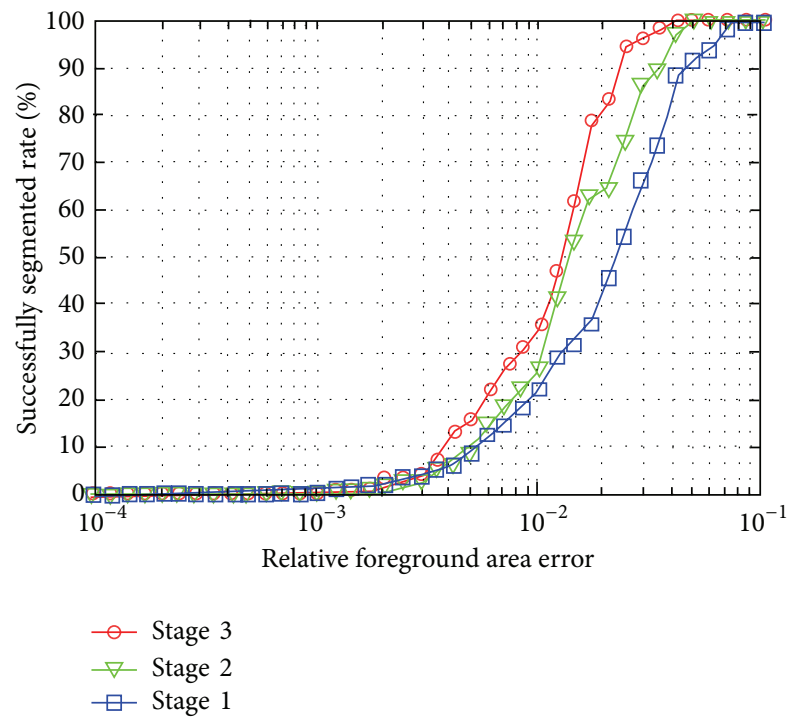

(c)

FIGURE 7: Successfully segmented rate versus (a) false positive rate, (b) misclassification error, and (c) relative foreground area error.
2.00 GB of main memory to test the presented algorithm on iris image database, UBIRIS.v1. For an eye-image with size of $600 * 800$ pixels, the average execution time is 2.78 seconds and the mean value of accuracy is 0.9813 . Several measures of errors, such as ME, ACC, RFAE, and EMM, are conducted to measure the performance of the proposed algorithm. The experimental results show that the proposed algorithm is effective and efficient in iris segmentation and the proposed method has the following advantages: (i) iris boundary extraction is very accurate due to Delogne-Kåsa circle fitting, (ii) the algorithm is robust in illumination and large reflection areas and also in eyelid and eyelash occlusions because the noise detection is based on the statistical characteristics of the iris, eyelids, and eyelashes in each image, and (iii) the algorithm is less time-consuming because noise detection is operated only on the extracted iris region and not on whole eye-image. In the future we will develop a high performance iris recognition system based on accurate iris segmentation.

\section{Conflict of Interests}

The authors declare that there is no conflict of interests regarding the publication of this paper.

\section{Acknowledgment}

This research was supported by the National Science Council, Taiwan, under Grant NSC 102-2221-E-005-082.

\section{References}

[1] K. Delac and M. Grgic, "A survey of biometric recognition methods," in Proceedings of the 46th International Symposium Electronics in Marine (Elmar '04), pp. 184-193, June 2004.

[2] Y. Chen, M. Adjouadi, C. Han et al., "A highly accurate and computationally efficient approach for unconstrained iris segmentation," Image and Vision Computing, vol. 28, no. 2, pp. 261-269, 2010.

[3] J. Daugman, "The importance of being random: statistical principles of iris recognition," Pattern Recognition, vol. 36, no. 2, pp. 279-291, 2003.

[4] D. de Martin-Roche, C. Sanchez-Avila, and R. Sanchez-Reillo, "Iris-based biometric recognition using dyadic wavelet transform," IEEE Aerospace and Electronic Systems Magazine, vol. 17, no. 10 , pp. 3-6, 2002.

[5] T. A. Camus and R. Wildes, "Reliable and fast eye finding in close up images," in Proceedings of the IEEE 16th International Conference on Pattern Recognition, pp. 389-394, 2002.

[6] H. Proença and L. A. Alexandre, "Iris segmentation methodology for non-cooperative recognition," IEE Proceedings: Vision, Image and Signal Processing, vol. 153, no. 2, pp. 199-205, 2006.

[7] H. Proença and L. A. Alexandre, "Ubiris: a noisy iris image database," in Proceedings of the International Conference on Image Analysis and Processing, pp. 970-977, 2005.

[8] T. D’Orazio, C. Guaragnella, M. Leo, and A. Distante, "A new algorithm for ball recognition using circle Hough transform and neural classifier," Pattern Recognition, vol. 37, no. 3, pp. 393-408, 2004. 
[9] C. Kimme, D. Ballard, and J. Sklansky, "Finding circles by an array of accumulators," Communications of the ACM, vol. 18, no. 2, pp. 120-122, 1975.

[10] M. Ceccarelli, A. Petrosino, and G. Laccetti, "Circle detection based on orientation matching," in Proceedings of the 11th International Conference on Image Analysis and Processing, pp. 119-124, 2002.

[11] N. N. Chan, "On circular functional relationships," Journal of the Royal Statistical Society B, vol. 27, pp. 45-56, 1965.

[12] E. E. Zelniker and I. V. L. Clarkson, "A statistical analysis of the Delogne-Kåsa method for fitting circles," Digital Signal Processing, vol. 16, no. 5, pp. 498-522, 2006.

[13] N. Otsu, "A threshold selection method from gray-level histograms," IEEE Transactions on Systems, Man, and Cybernetics, vol. 9, no. 1, pp. 62-66, 1979.

[14] X. Zhang, Q. Wang, H. Zhu, C. Yao, L. Gao, and X. Liu, "Noise detection of iris image based on texture analysis," in Proceedings of the 2009 Chinese Control and Decision Conference (CCDC '09), pp. 2366-2370, June 2009.

[15] W.-K. Kong and D. Zhang, "Detecting eyelash and reflection for accurate iris segmentation," International Journal of Pattern Recognition and Artificial Intelligence, vol. 17, no. 6, pp. 10251034, 2003.

[16] J. Huang, Y. Wang, T. Tan, and J. Cui, "A new iris segmentation method for recognition," in Proceedings of the 17th International Conference on Pattern Recognition (ICPR '04), pp. 554-557, August 2004.

[17] W. Yuan and W. He, "A novel eyelash detection method for iris recognition," in Proceedings of the 2005 27th Annual International Conference of the Engineering in Medicine and Biology Society (IEEE-EMBS '05), pp. 6536-6539, September 2005.

[18] M.-H. Horng, "Performance evaluation of multiple classification of the ultrasonic supraspinatus images by using ML, RBFNN and SVM classifiers," Expert Systems with Applications, vol. 37, no. 6, pp. 4146-4155, 2010.

[19] M. Vatsa, R. Singh, and A. Noore, "Improving iris recognition performance using segmentation, quality enhancement, match score fusion, and indexing," IEEE Transactions on Systems, Man, and Cybernetics B: Cybernetics, vol. 38, no. 4, pp. 1021-1035, 2008. 


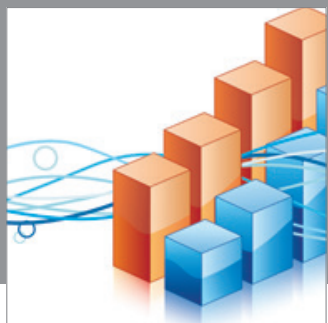

Advances in

Operations Research

mansans

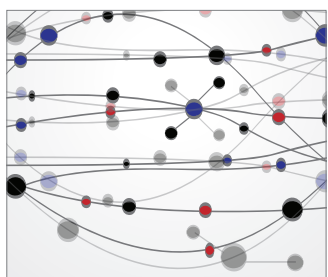

The Scientific World Journal
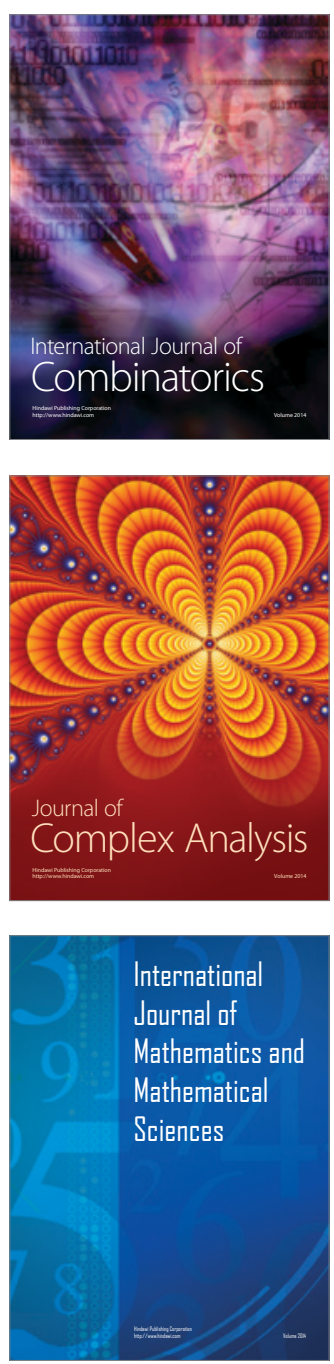
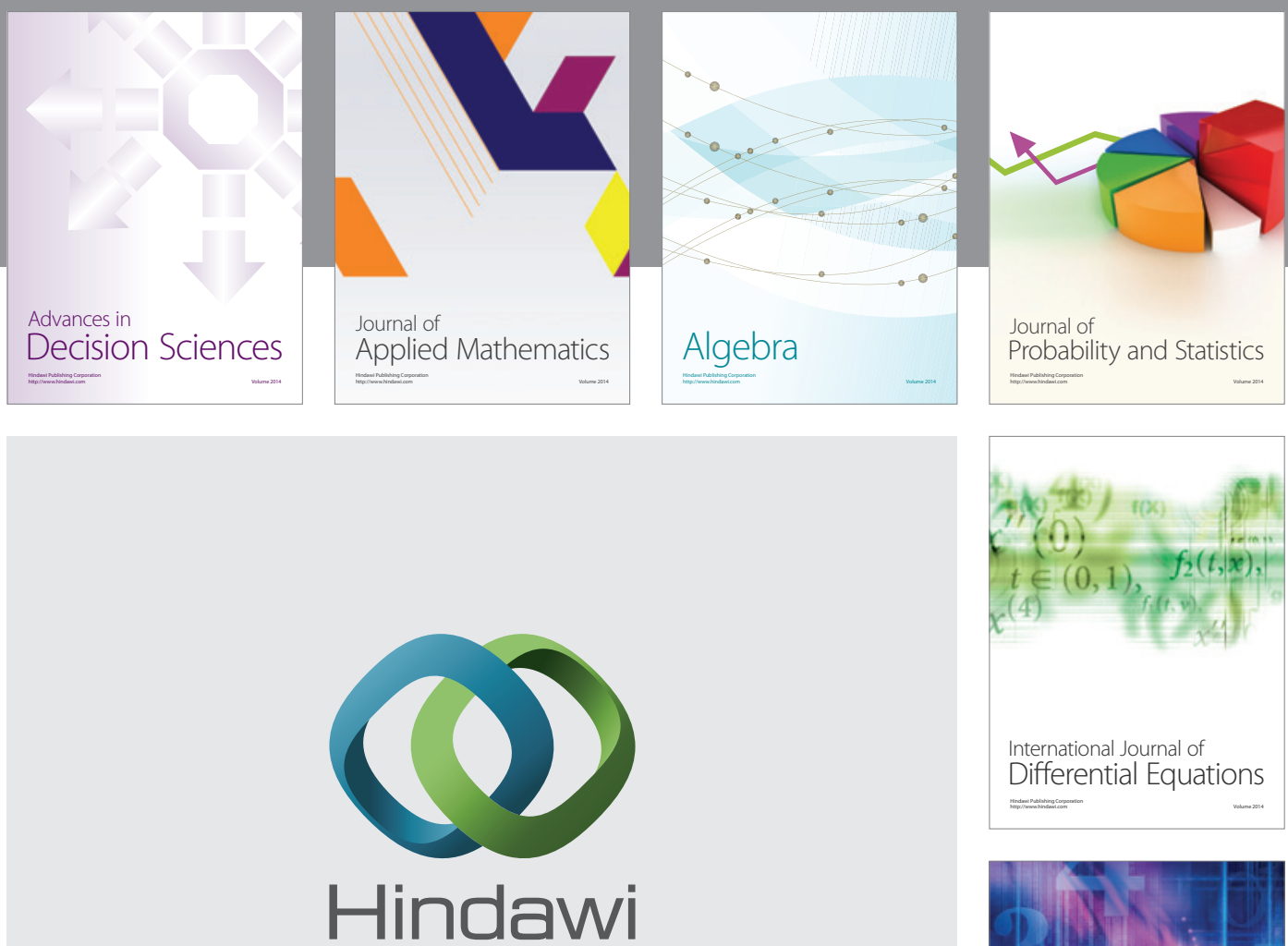

Submit your manuscripts at http://www.hindawi.com
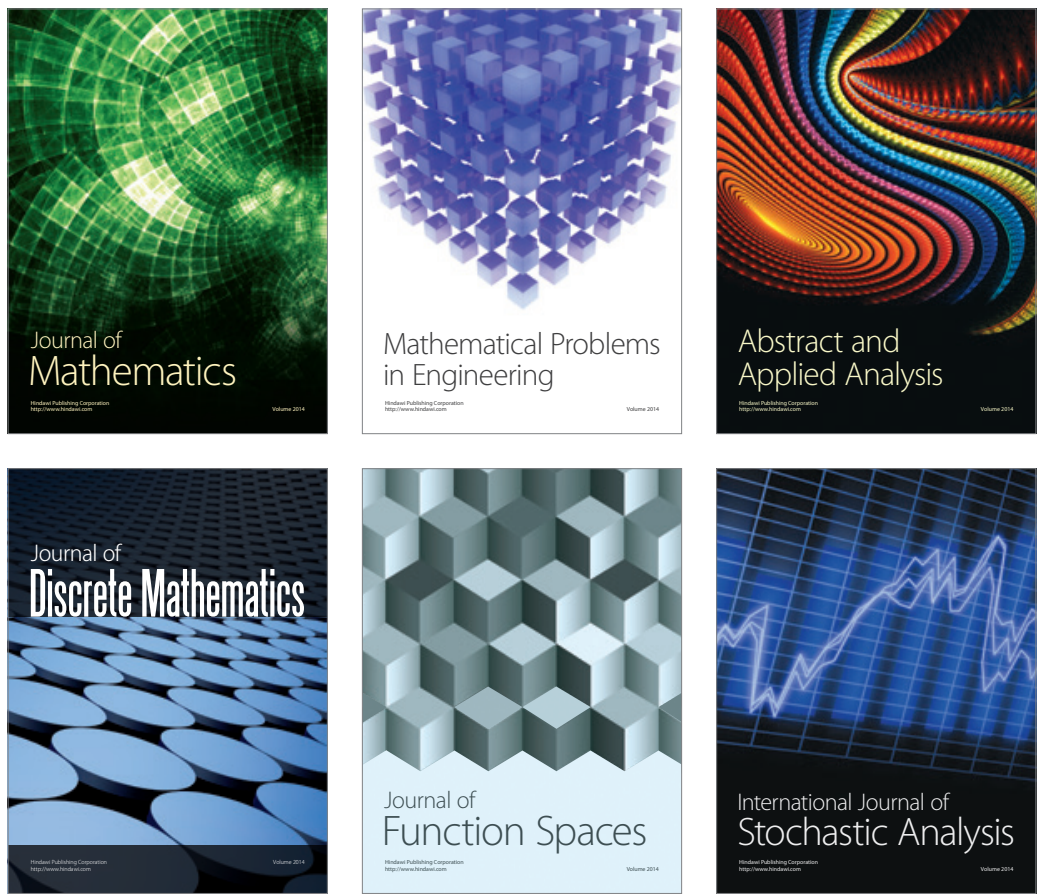

Journal of

Function Spaces

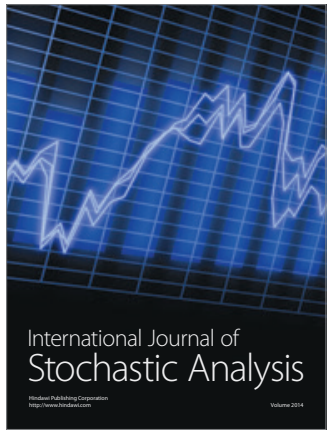

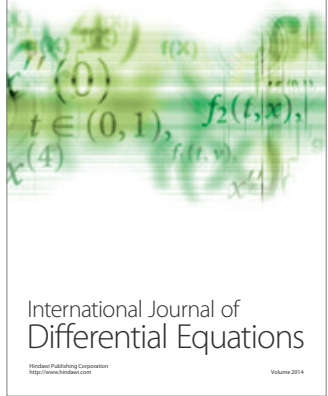
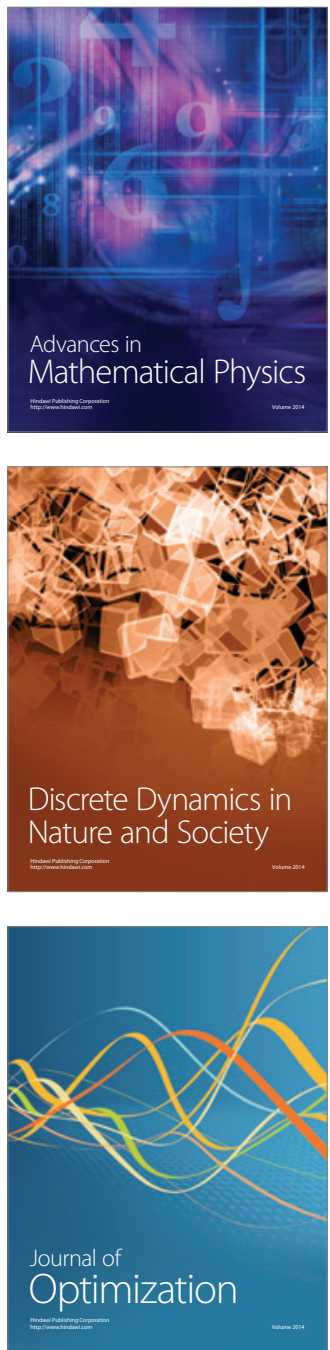